\title{
Morphology and Microstructural Characterization of Low-temperature Plasma Modified Bio-based Silica/carbon Using FE-SEM
}

\author{
Zaheeruddin Mohammed, Shaik Jeelani and Vijay Rangari \\ Tuskegee University, Tuskegee, Alabama, United States
}

Carbonaceous materials due to their inert nature and chemical/thermal stability have huge potential in the areas of environment and energy applications. Synthesis and development of carbon with high performance and multifunctionality has been the focus of researchers recently. Traditionally carbon materials are synthesized using materials, which are highly dependent on fossil fuels. Therefore, in order to develop a more sustainable carbon, researchers are considering synthesis of carbon from natural precursors $[1,2]$. Among natural precursors for carbon Rice Husk $(\mathrm{RH})$ is of particular importance due to presence of silica ( $\mathrm{SiO} 2)$ making it a naturally occurring organic-inorganic composite [3]. Carbon derived from sustainable sources is of limited use mainly due to inactive surface functionalities and reduced surface area. Activation of carbon materials is typically achieved through wet chemical processes with chemicals like $\mathrm{K} 2 \mathrm{CO} 3, \mathrm{H} 2 \mathrm{O} 2, \mathrm{HNO} 3, \mathrm{KOH}$ and $\mathrm{O} 2, \mathrm{LaCl} 3, \mathrm{H} 3 \mathrm{PO} 4, \mathrm{ZnCl} 2, \mathrm{CO} 2-\mathrm{NH} 3$ mixture, amine, methanol etc. The objective of activation is to increase surface area and introduce active functional groups. Nowadays, plasma technology is being regarded as a highly efficient tool for material preparation or modification [4]. Usually, plasma technology is a low energy, cost effective process. It also does not yield any harmful chemical waste making it a sustainable route for material modifications. Low-temperature plasmas (LTPs) are a unique state of matter due to the presence of neutral atoms and molecules, radicals, excited states, ions, and electrons. In a typical plasma material modification process, the energetic radicals attack the surface of material and then a series of physical and chemical reactions occur during the energy exchange, the redundant reactants then are removed from the material surface and thus change the physical and chemical properties of the material. [5]

In present study, Rice Husk (RH) was washed with distilled water and dried at $120^{\circ} \mathrm{C}$ for $24 \mathrm{~h}$ followed by ball milling for $3 \mathrm{~h}$. Thus, produced powder was taken into the closed vessel chamber furnace. The furnace was heated to $500{ }^{\circ} \mathrm{C}$ at the rate of $10{ }^{\circ} \mathrm{C} / \mathrm{min}$ ramp and then pyrolysis was carried out for $2 \mathrm{~h}$ under this condition. Obtained rice husk char (60\% yields) was then mortared gently to make rice husk ash (RHA). The RHA contains $52 \% \mathrm{CO} 2$ and $48 \% \mathrm{SiO} 2$ confirmed by TGA analysis in presence of Oxygen. The RHA was then surface treated with plasma of different gases argon (Ar), oxygen (O2) and sulfur hexafluoride (SF6) using 150 Watts power for a duration of $60 \mathrm{~min}$. SEM analysis was performed using FESEM (JOEL JSM-7200F) to study surface morphology and microstructure changes due to plasma treatments. Prior to testing carbon samples were sputter coated with gold-palladium for 5 min in Hummer 6.2 sputtering system purged with Argon gas and operated at $20 \mathrm{mTorr}, 5 \mathrm{~V}$, and $10 \mathrm{~mA}$.

FE-SEM micrographs revealed noticeable etching on the surface of silicon/carbon particles for oxygen and SF6 treatments. For argon plasma treatment there was no observable change in the morphology, mainly due to inert nature of Ar gas (Fig 1(b)). For oxygen treatments, highly energized oxygen ions from plasma readily reacted with surface carbon atoms to either etch the surface and form $\mathrm{CO} 2$ gas or functionalize the surface forming carbonyl or carboxyl groups (Fig 1(c)). In case of SF6 treatment, drastic etching was observed due to presence of fluoride ions during plasma treatments (Fig 1(d)). The silica particles were functionalized by forming S-OR groups. The micro pores formed due to plasma etching can be helpful in increasing active surface area of silica/carbon material by many orders. Similarly, surface functionalization of silica/carbon improves chemical activity of the materials with surrounding the materials. Thus, it can be concluded that plasma treatments are effective in altering surface morphology 
and microstructure of silica/carbon material derived from rice husk improving its suitability for environment, energy and storage applications.
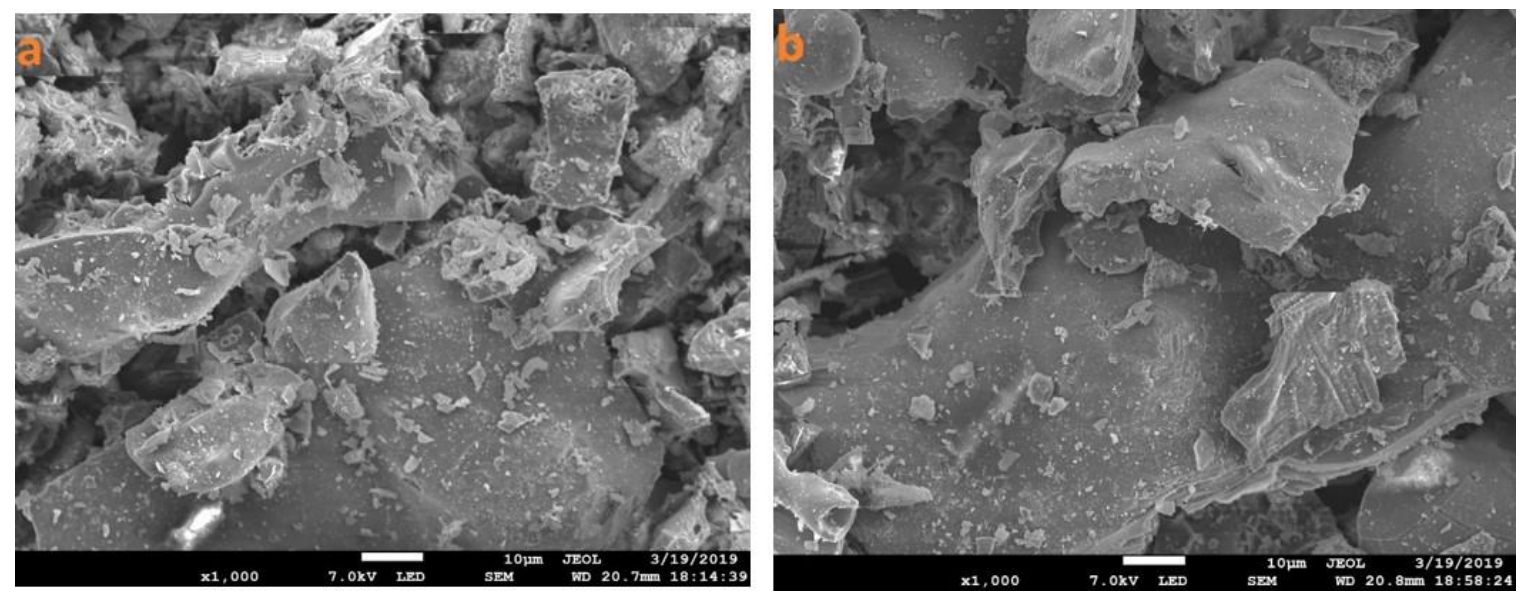

Figure 1. FE-SEM micrographs of silica/carbon treated with plasma Untreated (a), Ar (b),
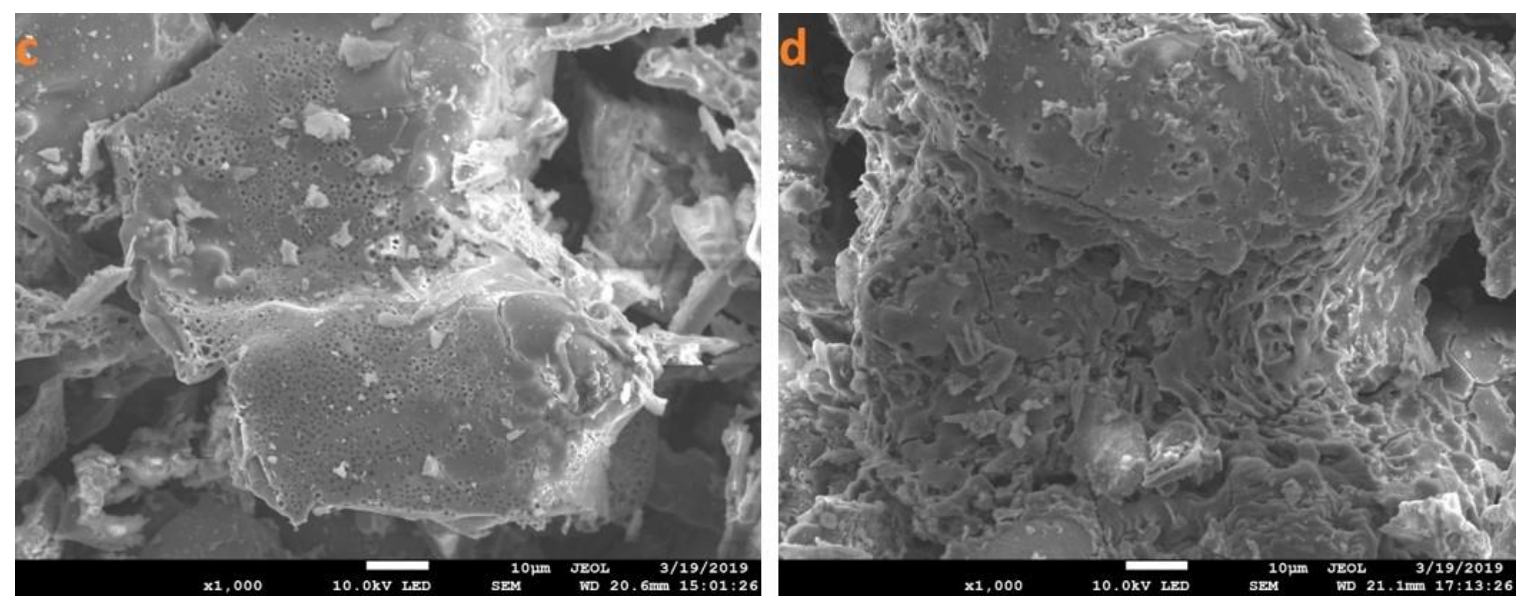

Figure 2. FE-SEM micrographs of silica/carbon treated with plasma $\mathrm{O}_{2}(\mathrm{c}) \mathrm{SF}_{6}(\mathrm{~d})$

\section{References}

[1] Zhang, Zhengping, et al. Advanced Materials 31.13 (2019): 1805718.

[2] Mohammed, Zaheeruddin, Shaik Jeelani, and Vijaya Rangari. Microscopy and Microanalysis 25.S2 (2019): 1112-1113.

[3] Zhang, Wenli, et al. Energy 128 (2017): 618-625.

[4] Mohammed, Zaheeruddin, Shaik Jeelani, and Vijaya Rangari. JOM 72.3 (2020): 1-10.

[5] Dou, Shuo, et al. Advanced materials 30.21 (2018): 1705850. 